\title{
Non-smooth geodesic flows and the earthquake flow on Teichmüller space*
}

\author{
HOWARD WEISS \\ Department of Mathematics, California Institute of Technology, Pasadena, \\ California 91125, U.S.A.
}

(Received 12 December 1986 and revised 30 March 1987)

Abstract. Thurston generalized the notion of a twist deformation about a simple closed geodesic on a hyperbolic Riemann surface to a twisting or shearing along a much more complicated object called a measure geodesic lamination. This new deformation is called an earthquake and it generates a flow on the tangent bundle of Teichmüller space.

In this paper we study the earthquake flow. We show that the flow is not smooth and that it is not the geodesic flow for an affine connection. We also derive the explicit form of the system of differential equations which earthquake trajectories satisfy.

\section{Introduction}

Thurston generalized the notion of a twist deformation about a simple closed geodesic on a hyperbolic Riemann surface to a twisting or shearing along a much more complicated object called a measured geodesic lamination. This new deformation is called an earthquake, and it generates a flow on the tangent bundle to Teichmüller space.

In this paper we study the dynamics of the earthquake flow. We show this flow is not smooth, and we derive the explicit form of the system of differential equations which earthquake trajectories (paths) satisfy.

Our strategy is to exploit the analogies between the earthquake flow and the geodesic flow on a Riemannian manifold. Along the way, we find sufficient conditions to determine when a flow on the tangent bundle of a smooth manifold is the geodesic flow for an affine connection. It turns out that the earthquake flow satisfies all of the conditions except for smoothness.

Since very little about measured geodesic laminations has appeared in print, we begin, for the readers convenience, with two short sections containing background material on hyperbolic geometry and measured geodesic laminations.

Most of the contents of this paper appears in the latter half of my thesis. I would like to thank my advisor, Scott Wolpert, for his consummate guidance and encouragement.

\footnotetext{
* Partially supported by Sloạ Foundation Doctoral Dissertation Fellowship.
} 


\subsection{Preliminaries on hyperbolic geometry}

Definition. A hyperbolic Riemann surface is a smooth oriented surface of genus $\mathrm{g} \geq 2$ equipped with a metric of constant curvature -1 .

By the uniformization theorem, every hyperbolic Riemann surface $R$ is isometric to a surface of the form $H^{2} / \Gamma$, where $H^{2}$ is the hyperbolic plane and $\Gamma$ is a Fuchsian group (a discrete subgroup of $\operatorname{PSL}(2, R)$ ) acting by isometries, and isomorphic to $\pi_{\mathrm{l}}(R)$. Let $\Sigma_{\mathrm{g}}$ denote a fixed smooth, compact, oriented surface of genus $\mathrm{g}$.

Definition. The Teichmüller space of genus $g, \mathscr{T}_{g}$, is the space of discrete and faithful representations from $\pi_{1}\left(\Sigma_{g}\right) \rightarrow \operatorname{PSL}(2, R)$ modulo conjugacy. Equivalently, $\mathscr{T}_{g}$ may be defined as the space of hyperbolic metrics on $\Sigma_{g}$ with two metrics identified if there is an isometry between them which is isotopic to the identity.

Every Mobius transformation $A$ acting on $H^{2}$ has a continuous extension to its boundary, denoted $S_{\infty}^{1}$, and called the 'circle at $\infty$ '. Pairs of distinct points on $S_{\infty}^{1}$ are in 1-1 correspondence with (oriented) geodesics in $H^{2}$. Every hyperbolic Mobius transformation $A(|\operatorname{tr} A|>2)$ has exactly two fixed points both on $S_{\alpha}^{1}$. The geodesic corresponding to the fixed points of $A \in \Gamma$ projects to the unique closed geodesic in $R$ contained in the free homotopy class of $i(A) \in \pi_{1}(\Sigma)$ under the isomorphism $i: \Gamma \rightarrow \pi_{1}(\Sigma)$.

Let $f$ be a homeomorphism between two Riemann surfaces $R_{1}$ and $R_{2}$ and let $f_{*}$ be the induced isomorphism between their uniformizing groups $\Gamma_{1}$ and $\Gamma_{2}$. This isomorphism gives a canonical 1-1 correspondence between elements of $\Gamma_{1}$ and $\Gamma_{2}$, and hence between closed geodesics on $R_{1}$ and $R_{2}$. Consequently, this isomorphism also induces a mapping between the sets of fixed points of elements of $\Gamma_{1}$ and $\Gamma_{2}$, which are dense in $S_{\infty}^{1}$.

The following theorem of Nielsen states that this mapping has a unique extension to a homeomorphism between the circles at infinity. This homeomorphism enables us to identify complete geodesics on the two surfaces.

Theorem. (Nielsen, [T].) Let $f: R_{1} \rightarrow R_{2}$ be a homeomorphism between two closed hyperbolic Riemann surfaces. Then any lift of $f$ to a homeomorphism $\tilde{f}: H^{2} \rightarrow H^{2}$ has a unique continuous extension $\tilde{f}$ to a homeomorphism of $H^{2} \cup S_{\propto}^{1}$, and the extension is invariant under isotopy, depending only on the lift and on $R_{1}$ and $R_{2}$. The extension is also equivariant so that the fixed points of any element $A_{1} \in \Gamma_{1}$ are taken to the fixed points of $A_{2} \in \Gamma_{2}$ where $\tilde{f}_{*}\left(A_{1}\right)=A_{2}\left(\tilde{f}_{*}: \Gamma_{1} \rightarrow \Gamma_{2}\right.$ induced by $\left.\tilde{f}\right)$.

A classical theorem of Morrey [AH] implies that $\tilde{f} \mid S_{\infty}^{1}$ is Holder continuous. However, an elementary argument of Sullivan shows that the equivariance prevents $\tilde{f} \mid S_{\infty}^{1}$ from being Lipschitz. The derivative of $\tilde{f} \mid S_{\infty}^{1}$ is a totally singular measure, hence $\tilde{f} \mid S_{\infty}^{1}$ is not absolutely continuous.

\subsection{Measured geodesic laminations}

Let $R$ be a closed hyperbolic Riemann surface of genus $g$.

Definition. A geodesic lamination $\mathscr{G}$ on $R$ is a foliation of a closed subset of $R$ by complete, simple geodesics. 
Let $\mathscr{A}$ denote the set whose elements are simple, smooth open arcs contained in $R$ which are transverse to $\mathscr{G}$, and have endpoints in $R-\mu$.

Definition. A Measured geodesic lamination (MGL) is a pair $(\mathscr{G}, \mu)$, where $\mathscr{G}$ is a geodesic lamination and $\mu$ is a transverse measure satisfying:

(1) support $(\mu)=\mathscr{G}$.

(2) If $t_{1}$ is homotopic to $t_{2}$ in $\mathscr{A}$, then $\mu\left(t_{1}\right)=\mu\left(t_{2}\right)$.

'Trivial' Examples of an $M G L$. Let $(\mathscr{G}, \mu)$ be a finite disjoint union of simple closed geodesics $\phi_{1}, \ldots, \phi_{n}$ on $R$ with counting measure $\mu=\sum_{k=1}^{n} a_{k} \delta_{\phi_{k}}, a_{k}>0$.

We will follow custom and denote the $\operatorname{MGL}(\mathscr{G}, \mu)$ by $\mu$. The following three theorems about MGLs are due to Thurston [see T]:

THEOREM. If $\mu$ is a MGL on $R$ and $t \in \mathscr{A}$, then $t \cap \mu$ is either a discrete set or the union of a Cantor set and a discrete set. Moreover, the isolated points of $t \cap \mu$ are exactly the intersections of $t$ with simple closed leaves of $\mu$.

Theorem. If $\mu$ is a MGL on $R$, then Hyperbolic Area $(\mu)=0$.

THEOREM. If $\mu$ is a MGL on $R$, then $R-\mu$ is a finite union of ideal polygons and Riemann surfaces with geodesic boundary.

The transverse measure on a MGL $\mu$ induces a Lebesgue-Stiltjes measure on arcs contained in $\mathscr{A}$. This allows us to integrate functions along transverse arcs. For a transverse arc $t \in \mathscr{A}$, let $\theta$ be the angle that the leaves of $\mu$ make with $t$, measured counterclockwise from $t$ to $\mu$. It is easy to see that $\theta$ is a Lipschitz function along $t\left[W_{1}\right]$, and therefore may be integrated with respect to $d \mu$. We define the total angle and total cosine of an arc $t \in \mathscr{A}$ by:

$$
\theta(t, \mu)=\int_{t} \theta d \mu, \quad \cos (t, \mu) \equiv \int_{t} \cos \theta d \mu .
$$

Similarly, we define the total angle and total cosine of a simple closed geodesic $\phi$ by:

$$
\theta(\phi, \mu) \equiv \int_{\phi} \theta d \mu, \quad \cos (\phi, \mu) \equiv \int_{\phi} \cos \theta d \mu .
$$

Let $M L(=M L(R))$ denote the set of $M G L s$ on $R$. Thurston defined the following topology on $M L$ : Given a finite set of arcs $\left\{t_{k}\right\}_{k=1}^{n} \subset \mathscr{A}, \varepsilon>0$, and $\mu \in M L$, a

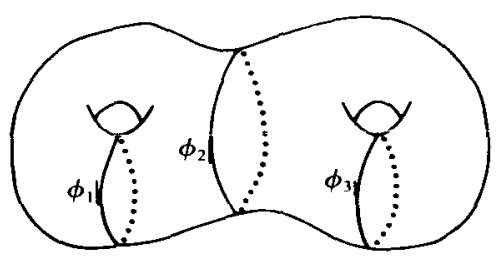

Figure 1. 'Trivial MGL'. 
neighborhood basis of $\mu$ is given by:

$$
\begin{aligned}
\mathscr{B}\left(\mu,\left\{t_{k}\right\}_{k=1}^{n}, \varepsilon\right) & =\left\{\nu \in M L:\left|\left(\mu\left(t_{k}\right), \theta\left(t_{k}, \mu\right)\right)-\left(\nu\left(t_{k}\right), \theta\left(t_{k}, \nu\right)\right)\right|\right. \\
& <\varepsilon \quad k=1,2, \ldots, n\} .
\end{aligned}
$$

The following theorem of Thurston may be thought of as a strong existence statement for MGLs:

THEOREM. (Thurston, [T].) ML is homeomorphic to $\mathbb{R}^{6 g-6}$.

Let $\mathscr{S}$ denote the set of simple closed geodesics on $R$. We can embed $\mathscr{S} \times \mathbb{R}^{+}$in $M L$ by sending $(\phi, r)$ to the MGL consisting of $\phi$ with $\mu=r \cdot \delta_{\phi}$.

ThEOREM. (Thurston, [T]). $\mathscr{S} \times \mathbb{R}^{+}$is dense in $M L$.

This theorem allows one to extend notions defined for simple closed geodesics to MGLs.

Given two Riemann surfaces $R_{1}$ and $R_{2}$ of genus $g$, along with an isomorphism between their uniformizing groups, we have seen that the mapping between their circles at infinity may be used to transport and/or identify MGLs on the two Riemann surfaces. The following two theorems are due to Thurston [T].

(A) If $R_{1}$ and $R_{2} \in \mathscr{T}_{g}$, then $M L\left(R_{1}\right)$ is homeomorphic to $\operatorname{ML}\left(R_{2}\right)$.

(B) Suppose $R_{1}$ and $R_{2} \in \mathscr{T}_{g}$, thought of as hyperbolic structures on a fixed surface $\Sigma_{g}$. Let $\mu_{R_{1}}$ be a MGL on $R_{1}$ and let $\mu_{R_{2}}$ be the 'corresponding' MGL on $R_{2}$. Then the leaves of $\mu_{R_{1}}$ are isotopic to the corresponding leaves of $\mu_{R_{2}}$ on $\Sigma_{g}$.

2. Geodesic flows with low regularity, ODEs, and affine connections

In this section we find sufficient conditions to determine when a flow on the tangent bundle of a smooth manifold is the geodesic flow for an affine connection. In addition, we prove a structure theorem for flows which satisfy all of the conditions except for smoothness. We will later show that the earthquake flow is of this type. The reader is advised to keep in mind that the results in this section are completely independent of any metric structure on $M$.

Let $M$ be a $C^{\infty}$ manifold.

Definition. A mapping $\phi: \mathbb{R} \times M \rightarrow M$ is called a global flow on $M$ if for all $x \in M$, and $s, t \in \mathbb{R}$, we have

(1) $\phi(0, x)=x$

(2) $\phi(t, \phi(s, x))=\phi(t+s, x)$.

We can impose regularity on the flow by requiring $\phi$ to be $C^{r} 0 \leq r \leq \omega$, Lipschitz, Hölder continuous, etc. Unless otherwise stated all flows will be assumed to be global $C^{0}$ (topological) flows.

Definition. If $\phi$ is a flow on $M$, and $x \in M$, the curve

$$
\begin{aligned}
c_{x}: \mathbb{R} & \rightarrow M, \\
t & \rightarrow \phi(t, x)
\end{aligned}
$$

is called the trajectory of $x$. 
Definition. Let $\phi$ be a flow on $M$ such that every trajectory of $\phi$ is a $C^{\infty}$ curve in $M$, i.e., $\phi$ is a $C^{\infty}$ function in its first variable $t$. Then the vector field

$$
\begin{aligned}
\Phi: M & \rightarrow T M \\
x & \left.\rightarrow \frac{d}{d t}\right|_{t=0} \phi(t, x)=c_{x}(0)
\end{aligned}
$$

is called the infinitesimal generator of $\phi$.

We shall refer to such a flow as a flow with smooth trajectories. By definition, any such flow $\phi$ satisfies the first order ODE:

on $M$.

$$
\Phi(x)=\left.\frac{d}{d t}\right|_{t=0} \phi(t, x)
$$

Definition. $\phi$ has the Affine Reparametrization (AR) Property if whenever $c(t)$ is a trajectory for $\phi, c(a t+b)$ is also a trajectory for $\phi$, for all $a, b \in \mathbb{R}^{+}$.

Since $M$ is a $C^{\infty}$ manifold, its tangent bundle, $T M$, is also a $C^{\infty}$ manifold, and hence we can study flows on $T M$. From now on we will restrict our study to flows on $T M$.

Let $\phi$ be a global flow on $T M$ with smooth trajectories and let $\Phi$ be its infinitesimal generator.

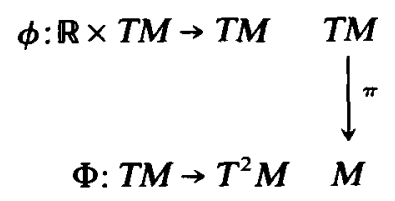

Definition. $\phi$ has the Second Order ODE (SODE) Property if

$$
\phi(t, x)=\frac{d}{d t}(\pi \circ \phi(t, x)) \quad \text { for all } x \in T M, \quad t \in \mathbb{R} .
$$

SODE is the condition that each trajectory of $\phi$, considered as a section of $\pi: T M \rightarrow$ $M$, is precisely the velocity vector field of the projection to $M$.

Suppose $c(t)$ is a trajectory of $\phi$ and let $\sigma(t)$ be its projection onto $M$. The SODE property implies $c(t)=\dot{\sigma}(t)$. Since $c(t)$ is a trajectory of $\phi$,

$$
\dot{c}(t)=\Phi(c(t))
$$

This may be rewritten as

$$
\ddot{\sigma}(t)=\Phi(\dot{\sigma}(t))
$$

Hence, if $\left(x_{1}, \ldots, x_{n}\right)$ are local coordinates in some open set $U \subset M$, then $\sigma(t)$ satisfies a system of second order quasilinear ODEs of the following type:

$$
\ddot{\sigma}_{k}(t)+R_{k}(\sigma(t), \dot{\sigma}(t))=0 \quad k=1,2, \ldots n .
$$

THEOREM 2.1. Let $\phi: \mathbb{R} \times T M \rightarrow T M$ be a global flow with smooth trajectories having the $A R$ and $S O D E$ properties. Then

$$
R_{k}(\sigma(t), \dot{\sigma}(t))=\Gamma_{i j}^{k}(\sigma(t), \dot{\sigma}(t)) \dot{\sigma}_{i}(t) \dot{\sigma}_{j}(t) \quad k=1,2, \ldots, n
$$

where the $\left\{\Gamma_{i j}^{k}\right\}$ are homogeneous functions of degree 0 in $\dot{\sigma}(t)$. 
If in addition the $\left\{R_{k}\right\}$ (hence the $\left\{\Gamma_{i j}^{k}\right\}$ ) are $C^{2}$ functions, then

$$
R_{k}(\sigma(t), \dot{\sigma}(t))=\Gamma_{i j}^{k}(\sigma(t)) \dot{\sigma}_{i}(t) \dot{\sigma}_{j}(t), \quad k=1,2, \ldots, n .
$$

Proof. We need the following lemma on homogeneous functions of degree 2.

LEMMA 2.2. If $f: \mathbb{R}^{n} \rightarrow \mathbb{R}$ is homogeneous of degree 2, i.e.

$$
f(a x)=a^{2} f(x), \text { for all } a \in \mathbb{R}^{+}, x \in \mathbb{R}^{n} \text {, then }
$$

(i) If $f \in C^{2}$, then $f$ is a quadratic form.

$$
f(x)=Q(x)=\sum_{i, j} c_{i j} x_{i} x_{j}
$$

(ii) With no smoothness condition on $f$,

$$
f(x)=Q(x) \cdot f\left(\frac{x}{|x|}\right)
$$

where $Q(x)$ is a quadratic form.

Proof of Lemma. (1) Since $a^{2} f(x)=f(a x)$, taking the second partial derivative with respect to $a$, we obtain:

$$
2 f(x)=\sum_{i, j} \frac{\partial^{2} f(a x)}{\partial\left(a x_{i}\right) \partial\left(a x_{j}\right)} x_{i} x_{j}
$$

The left hand side is independent of $a$, hence we conclude that $\partial^{2} f(x) / \partial x_{j} \partial x_{j}$ must be equal to a constant $c_{i j}$ for all $i, j$. Hence, $f(c)=\frac{1}{2} \sum_{i, j} c_{i j} x_{i} x_{j}$.

(2) Using $n$-dimensional polar coordinates, we write

$$
\left.x_{k}=r \cdot h_{k}\left(\frac{x_{1}}{r}, \ldots, \frac{x_{n-1}}{r}\right)=r \cdot h_{k}\left(\theta_{1}, \theta_{2}, \ldots, \theta_{n-1}\right)\right), \quad r=\sqrt{\sum_{k=1}^{n} x_{k}^{2}}=|x| .
$$

Then $f(x)=f\left(r \cdot h_{1}\left(\theta_{1}, \ldots, \theta_{n-1}\right), \ldots, r \cdot h_{n}\left(\theta_{1}, \ldots, \theta_{n-1}\right)\right)$. By hypotheses

$$
\begin{aligned}
& f(x)=r^{2} \cdot f\left(h_{1}\left(\theta_{1}, \ldots, \theta_{n-1}\right), \ldots, h_{n}\left(\theta_{1}, \ldots, \theta_{n-1}\right)\right) \\
& f(x)=Q(x) \cdot f\left(\frac{x_{1}}{r}, \ldots, \frac{x_{n}}{r}\right)=Q(x) \cdot f\left(\frac{x}{|x|}\right) .
\end{aligned}
$$

Proof of Theorem 2.1. Let $s=a t+b, a, b>0$. Since

$$
R_{k}\left(\sigma(s), a \frac{d \sigma(s)}{d s}\right)=R_{k}\left(\sigma(s), \frac{d \sigma(s)}{d t}\right)
$$

the $A R$ condition implies that this is equal to

$$
-\frac{d^{2} \sigma_{k}}{d t^{2}}(s)=-a^{2} \frac{d^{2} \sigma_{k}}{d s^{2}}(s)=a^{2} R_{k}\left(\sigma(s), \frac{d \sigma(s)}{d s}\right) .
$$

Hence, the $\left\{R_{k}\right\}$ are homogeneous of degree 2 in $\dot{\sigma}(t)$. The result follows from Lemma 2.2

It is convenient to use $(q, p)$ coordinates on $T M$. Let $(U, x)$ be a chart for $M$, and define

$$
\begin{aligned}
& q_{k}=x_{k} \circ \pi \quad k=1, \ldots, n . \\
& p_{k}=d x_{k}
\end{aligned}
$$


Then $\left(\pi^{-1}(U),(q, p)\right)$ is a chart for $T M$. Any vector field $X$ on $T M$ may be written locally (in this chart) as

$$
X=\sum_{k=1}^{n} f_{k}(q, p) \frac{\partial}{\partial q_{k}}+\sum_{k=1}^{n} g_{k}(q, p) \frac{\partial}{\partial p_{k}} .
$$

LEMMA 2.3. Let $\phi: \mathbb{R} \times T M \rightarrow T M$ be a global flow with smooth trajectories having the $A R$ and SODE properties, and let $\Phi$ be its infinitesimal generator. Then in local coordinates,

$$
\Phi=\sum_{k=1}^{n} p_{k} \frac{\partial}{\partial q_{k}}+\sum_{k=1}^{n}\left(-\Gamma_{i j}^{k}(q, p) p_{i} p_{j}\right) \frac{\partial}{\partial p_{k}} .
$$

Proof. Follows immediately from Theorem 2.1 and definitions.

Corollary 2.4. Suppose $\phi$ as in previous lemma. Then

$$
\Phi \in C^{r} \Rightarrow \phi \in C^{r}, \text { for } r \geq 2 \text {. }
$$

Proof. By the preceding lemma, if $\Phi \in C^{r}, r \geq 2$, then the $\left\{\Gamma_{i j}^{k}\right\} \in C^{r}$. It immediately follows from the "smooth dependence upon initial parameters" theorem from ODEs [CL] along with Theorem 2.1, that $\phi \in C^{r}$.

Proposition 2.5. In Theorem 2.1, the $\left\{\Gamma_{i j}^{k}\right\}$ transform as Christoffel symbols.

Proof. The proof is by explicit calculation and is omitted.

It is well known in differential geometry [ML, GKM], that any collection of $C^{s}$, $2 \leq s \leq \omega$ functions $\left\{\Gamma_{i j}^{k}\right\}$ on $M$ which transform as Christoffel symbols, induces a $C^{s}$ affine connection on $M$.

THEOREM 2.6. Let $\phi: \mathbb{R} \times T M \rightarrow T M$ be a global flow with smooth trajectories having the $A R$ and SODE properties and let $\Phi$ be the infinitesimal generator of $\phi$. Assume $(U, x)$ is a chart for $M$. Then

(a) The trajectories of $\phi$ are solutions to the following systems of ODEs:

$$
\ddot{x}_{k}+\Gamma_{i j}^{k}(x, \dot{x}) \dot{x}_{i} \dot{x}_{j}=0, \quad k=1,2, \ldots, n .
$$

(b) If $\Phi \in C^{r}, r \geq 2$, then the trajectories are solutions to:

$$
\ddot{x}_{k}+\Gamma_{i j}^{k}(x) \dot{x}_{i} \dot{x}_{j}=0, \quad k=1, \ldots, n
$$

and $\phi \in C^{r}$.

(c) In cases (a) and (b), the $\left\{\Gamma_{i j}^{k}\right\}$ transform as Christoffel symbols. In case (a), the Christoffel symbols "live" on TM and not $M$ as one usually encounters in Riemannian geometry.

(d) In case (b), $\phi$ induces a symmetric $C^{r}$ affine connection $\nabla$ on $M$, and $\phi$ is the geodesic flow for $\nabla$.

Example. The geodesic flow on $T M$ for a smooth Riemannian metric induces the Levi-Civita connection on $M$.

Remark. With trivial modifications, all the results in this section apply to semiflows 
on $T M$. A semi-flow is a mapping $\phi: \mathbb{R}^{+} \times T M \rightarrow T M$ which satisfies the usual flow conditions.

\subsection{Earthquakes}

In [FN], Fenchel and Nielsen define the following 'twist deformation' of a compact Riemann surface $R$ : Choose any simple closed geodesic $\phi$. Cut $R$ open along $\phi$ to obtain a (possibly disconnected) Riemann surface with geodesic boundary. Now glue the boundary components back with a left twist of distance $t$, obtaining a new $R_{t}$.
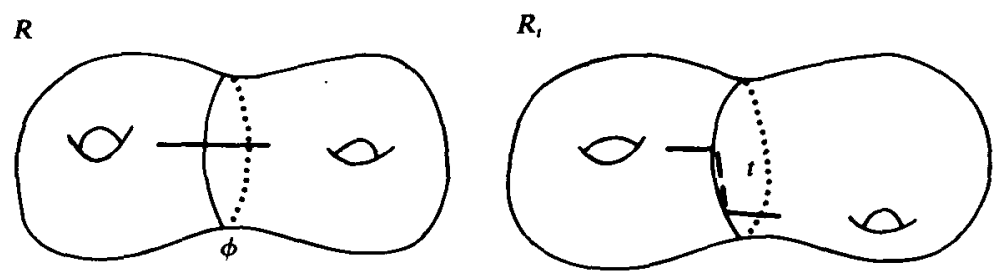

FIGURE 2

There exists a mapping $f_{t}: R \rightarrow R_{t}$, which is an isometry off of $\phi$, and not defined on $\phi$. For $R_{t}$ to determine a new point in $\mathscr{T}_{\mathrm{g}}$, not just moduli space, we require a homotopy class of maps from $R$ to $R_{t}$. This is done by requiring that any closed curve $\gamma$ in $R$ which intersects $\phi$ be mapped to a curve in $R_{t}$ homotopic to the following curve: $f_{t}(\gamma)$ until it hits $\phi$, then run along $\phi$ a distance $t$ to the left, then follow $f_{t}(\gamma) \cdots$. Thus, the Riemann surface obtained by a full left twist of $R$ is distinct from $R$ in $\mathscr{T}_{g}$.

Thurston generalized the FN deformation to a 'twisting' or 'shearing' along a MGL.

Recall, that for $R$, the set $\mathscr{S} \times \mathbb{R}^{+}$consisting of weighted simple curved geodesics in $R$ is dense in $M L$. The twist along a MGL $\mu$ is defined by the limit in $\mathscr{T}_{g}$ of any sequence of FN twist deformations along weighted simple curved geodesics which converge to $\mu$ in $M L$.

Definition. ([T].) The left earthquake deformation of $R$ at time $t$ determined by $\mu \in M L$ is the limit in $\mathscr{T}_{g}$ of the time $t$ twist deformation of $R$ for any sequence $\left(\alpha_{i}, r_{i}\right)$ in $\mathscr{S} \times \mathbb{R}^{+}$converging to $\mu$ in $M L$. It will be denoted $E(R, \mu, t)$.

In $\left[\mathbf{K}_{1}\right]$, Kerchoff shows that the definition is valid, i.e., that the limits exist and are independent of the approximating sequence and hence unique.

Remarks. (1) A FN twist deformation is an earthquake deformation and will be referred to as either a simple earthquake or a twist deformation.

(2) Earthquakes may be considered in several different ways: as deformations of Riemann surfaces, as maps from $\mathscr{T}_{g}$ to itself, or as paths in $\mathscr{T}_{g}$ as $t$ varies. We will usually view earthquake as paths in $\mathscr{T}_{g}$.

The following theorem of Kerckhoff tells us we can view $M L(R)$ as a non-linear model of $\left(\mathscr{T}_{g}\right)_{R}$ (the tangent space to $R$, viewed as a point of $\mathscr{T}_{g}$ ):

ThEOREM [Kerckhoff, $\mathbf{K}_{3}$ ]. Every tangent vector to $\mathscr{T}_{g}$ at a Riemann surface $R$ is 
tangent to a unique earthquake path in $\mathscr{T}_{\mathrm{g}}$ emanating from $R$. In fact:

$$
\begin{aligned}
\Psi: M L(R) & \rightarrow\left(\mathscr{T}_{g}\right)_{R} \\
\mu & \left.\rightarrow \frac{d}{d t}\right|_{t=0^{+}} E(R, \mu, t)
\end{aligned}
$$

is a homeomorphism.

It follows immediately from Kerckhoff's theorem that earthquake deformations give rise to a global (topological) semi-flow on $T \mathscr{T}_{g}$. Given a tangent vector to $\mathscr{T}_{g}$ at $R$, we first identify it with its unique MGL on $R$, and then earthquake along the MGL for time $t$. This defines a (parametrized) path in $\mathscr{T}_{g}$, and taking the velocity vector field along the path, a path in $T \mathscr{T}_{g}$ :

$$
\mathbb{R}^{+} \times\left(\mathscr{T}_{\mathrm{g}}\right)_{R} \stackrel{\Psi}{\rightarrow} \mathbb{R}^{+} \times M L(R) \stackrel{E}{\rightarrow} T \mathscr{T}_{\mathrm{g}}
$$

From this description, it is clear that the flow has the SODE property.

It is also easy to see that this flow has the $A R$ property, for suppose $E(R, \mu, t)$ is an earthquake path and $c>0$. Then $E(R, \mu, c \cdot t)=E(R, c \cdot \mu, t)$.

THEOREM. [Kerckhoff, $\mathbf{K}_{2}$ ]. Earthquake trajectories are real analytic paths in $\mathscr{T}_{\mathrm{g}}$, i.e., the earthquake flow $E$ is a real analytic function of time $t$.

These two theorems of Kerckhoff, along with Theorem 2.1, Proposition 2.5 and the observations above yield the following result:

THEOREM 2.7. Earthquake paths (trajectories of E) are solutions to the system of second order quasilinear ordinary differential equations:

$$
\ddot{x}_{k}+\Gamma_{i j}^{k}(x, \dot{x}) \dot{x}_{i} \dot{x}_{j}=0 \quad k=1, \ldots, 6 g-6,
$$

where the $\left\{\Gamma_{i j}^{k}\right\}$ transform as Christoffel symbols but live on $T \mathscr{T}_{g}$.

Let $\mathscr{E}$ be the infinitesimal generator of the earthquake flow.

$$
\mathscr{E}: T \mathscr{T}_{g} \rightarrow T^{2} \mathscr{T}_{\mathrm{g}}
$$

THEOREM 2.8 .

(a) $\mathscr{E}$ is not $C^{2}$ for $T \mathscr{T}_{\text {once punctured torus. }}$

(b) $\mathscr{E}$ is not $C^{2}$ for $T \mathscr{T}_{\text {once holed torus. }}$

(c) $\mathscr{E}$ is not $C^{2}$ for $T \mathscr{T}_{g}$.

Hence, the earthquake flow is not a $C^{3}$ (smooth) flow!

The proof of Theorem 2.8 is by contradiction. To show (a), we first assume $\mathscr{E}$ is $C^{2}$. In that case, Theorems $2.6(\mathrm{~b})$ and 2.7 tells us that the flow is given by an affine connection, hence the Christoffel symbols are $\dot{x}$ independent. We then explicitly solve for the Christoffel symbols, and show that certain solutions of the resulting ODE cannot be earthquakes, for they fail to have a requisite convexity property of earthquake paths found by Kerckhoff. The proof of (b) is identical to (a), and (c) follows immediately from (b). 
Proof of $(a)$. Let $\mathscr{T}_{1,1}$ denote the Teichmüller space for the once punctured torus. It is well known [FN] that the geodesic length functions corresponding to the free homotopy classes of $\alpha$ and $\beta, l_{\alpha}$ and $l_{\beta}$, serve as local coordinates in $\left\{R \in \mathscr{T}_{1,1}: \alpha\right.$ is not perpendicular to $\beta\}$ or equivalently [WW] $\left\{R \in \mathscr{T}_{1,1}: l_{\beta}(R)>\right.$ $\left.2 \operatorname{coth}^{-1}\left(\cosh \left(l_{\alpha}(R) / 2\right)\right)\right\}$.

Assume $\mathscr{E}$ is $C^{2}$. Then by Theorem $2.6(\mathrm{~b})$ and 2.7 , all earthquake paths are solutions of the following system of ODSs:

$$
\begin{aligned}
& \ddot{x}_{1}+\Gamma_{i j}^{1}\left(x_{1}, x_{2}\right) \dot{x}_{i} \dot{x}_{j}=0 \\
& \ddot{x}_{2}+\Gamma_{i j}^{2}\left(x_{1}, x_{2}\right) \dot{x}_{i} \dot{x}_{j}=0 .
\end{aligned}
$$

To be precise, let $E(t)$ be an earthquake path, and let

$$
l_{\alpha}(t)=l_{\alpha} \circ E(t), \quad l_{\beta}(t)=l_{\beta} \circ E(t), \quad l(t)=\left(l_{\alpha}(t), l_{\beta}(t)\right) .
$$

The equations may be written as:

$$
\begin{aligned}
& \ddot{l}_{\alpha}(t)+\left[\Gamma_{\alpha \alpha}^{\alpha}(l(t)) \dot{i}_{\alpha}^{2}(t)+\Gamma_{\alpha \beta}^{\alpha}(l(t)) \dot{l}_{\alpha}(t) \dot{i}_{\beta}(t)+\Gamma_{\beta \beta}^{\alpha}(l(t)) \dot{i}_{\beta}^{2}(t)\right]=0 \\
& \ddot{l}_{\beta}(t)+\left[\Gamma_{\alpha \alpha}^{\beta}(l(t)) \dot{i}_{\alpha}^{2}(t)+\Gamma_{\alpha \beta}^{\beta}(l(t)) \dot{l}_{\alpha}(t) \dot{i}_{\beta}(t)+\Gamma_{\beta \beta}^{\beta}(l(t)) \dot{i}_{\beta}^{2}(t)\right]=0 .
\end{aligned}
$$

Notation. Let $\tau_{\alpha} l_{\beta}$ denote the derivative of the geodesic length function $l_{\beta}$ with respect to twisting (at unit speed) along (the geodesic freely homotopic to) $\alpha$.

Wolpert $\left[W_{2}\right]$ has found the following formulas for $\tau_{\alpha} l_{\beta}$ and $\tau_{\alpha}^{2} l_{\beta}$ :

$$
\begin{gathered}
\tau_{\alpha} l_{\beta}(R)=\sum_{p \in \alpha * \beta} \cos \theta_{p}, \\
\tau_{\alpha}^{2} l_{\beta}(R)=\sum_{(p, q) \in(\alpha * \beta) \times(\alpha * \beta)} \frac{e^{l_{1}}+e^{l_{2}}}{2\left(e^{l_{\beta}}-1\right)} \sin \theta_{p} \sin \theta_{q},
\end{gathered}
$$

where $\theta_{p}$ is the angle of $p$ measured from $\alpha$ to $\beta, 0 \leq \theta_{p} \leq \pi$. Given $(p, q) \epsilon$ $(\alpha \# \beta) \times(\alpha \# \beta), p, q$ divide $\beta$ into two subarcs, $l_{1}$ and $l_{2}$ are the lengths of these subarcs on $R$.

We will evaluate (1) and (2) along earthquakes $\alpha, \beta$ and $\alpha \beta$ and solve for the Christoffel symbols.

Twisting (earthquaking) along $\alpha$, equation (1) becomes:

$$
\tau_{\alpha}^{2} l_{\alpha}+\left[\Gamma_{\alpha \alpha}^{\alpha}\left(\tau_{\alpha} l_{\alpha}\right)^{2}+\Gamma_{\alpha \beta}^{\alpha}\left(\tau_{\alpha} l_{\alpha}\right)\left(\tau_{\alpha} l_{\beta}\right)+\Gamma_{\beta \beta}^{\alpha}\left(\tau_{\alpha} l_{\beta}\right)^{2}\right]=0 .
$$

$\tau_{\alpha} l_{\alpha}=0$ since $l_{\alpha}$ is constant along the path, hence $\Gamma_{\beta \beta}^{\alpha}=0$.

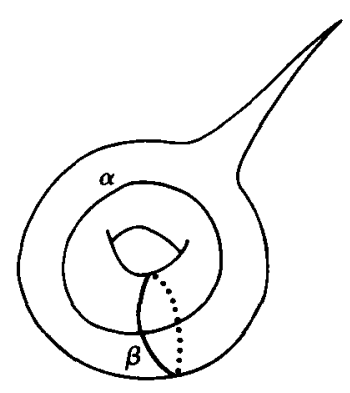

FIGURE 3 
Twisting along $\beta$, equation (1) becomes:

$$
\tau_{\beta}^{2} l_{\alpha}+\left[\Gamma_{\alpha \alpha}^{\alpha}\left(\tau_{\beta} l_{\alpha}\right)^{2}+\Gamma_{\alpha \beta}^{\alpha}\left(\tau_{\beta} l_{\alpha}\right)\left(\tau_{\beta} l_{\beta}\right)\right]=0
$$

$\tau_{\beta} l_{\beta}=0$ since $l_{\beta}$ is constant along the path.

Twisting along $\alpha \beta$, equation (1) becomes:

$$
\tau_{\alpha \beta}^{2} l_{\alpha}+\left[\Gamma_{\alpha \alpha}^{\alpha}\left(\tau_{\alpha \beta} l_{\alpha}\right)^{2}+\Gamma_{\alpha \beta}^{\alpha}\left(\tau_{\alpha \beta} l_{\alpha}\right)\left(\tau_{\alpha \beta} l_{\beta}\right)\right]=0 .
$$

Solving for $\Gamma_{\alpha \alpha}^{\alpha}$ and $\Gamma_{\alpha \beta}^{\alpha}$ in (4) and (5) we obtain:

$$
\Gamma_{\alpha \alpha}^{\alpha}=-\frac{\tau_{\beta}^{2} l_{\alpha}}{\left(\tau_{\beta} l_{\alpha}\right)^{2}} \quad \Gamma_{\alpha \beta}^{\alpha}=\frac{-\tau_{\alpha \beta}^{2} l_{\alpha}+\left(\frac{\tau_{\beta}^{2} l_{\alpha}}{\left.\tau_{\beta} l_{\alpha}\right)^{2}}\right)\left(\tau_{\alpha \beta} l_{\alpha}\right)^{2}}{\left(\tau_{\alpha \beta} l_{\alpha}\right)\left(\tau_{\alpha \beta} l_{\beta}\right)}
$$

Since $\operatorname{card}(\alpha \# \beta)=\operatorname{card}(\alpha \# \alpha \beta)=\operatorname{card}(\beta \# \alpha \beta)=1$, Wolpert's formulas reduce to (with the obvious notation):

$$
\begin{aligned}
& \tau_{\alpha} l_{\beta}=\cos _{\alpha, \beta}, \quad \tau_{\alpha}^{2} l_{\beta}=\frac{e^{l_{\beta}}+1}{2\left(e^{l_{\beta}}-1\right)} \sin _{\alpha, \beta}^{2} \\
& \tau_{\beta} l_{\alpha}=\cos _{\beta, \alpha}, \quad \tau_{\beta}^{2} l_{\alpha}=\frac{e^{l_{\alpha}}+1}{2\left(e^{l_{\alpha}}-1\right)} \sin _{\beta, \alpha}^{2} \\
& \tau_{\alpha \beta} l_{\alpha}=\cos _{\alpha \beta, \alpha}, \tau_{\alpha \beta}^{2} l_{\alpha}, \quad=\frac{e^{l}+1}{2\left(e^{l}-1\right)} \sin _{\alpha \beta, \alpha}^{2} \\
& \tau_{\alpha \beta} l_{\beta}=\cos _{\alpha \beta, \beta}, \tau_{\alpha \beta}^{2} l_{\beta}, \quad=\frac{e^{l_{\beta}}+1}{2\left(e^{l_{\beta}}-1\right)} \sin _{\alpha \beta, \beta}^{2} .
\end{aligned}
$$

Substituting these formulas into (6) and applying simple trigonometric identities, we obtain:

$$
\begin{aligned}
& \Gamma_{\alpha \alpha}^{\alpha}=-\frac{1}{2}\left(\frac{e^{l_{\alpha}}+1}{e^{l_{\alpha}}=1}\right) \tan _{\beta, \alpha}^{2} \\
& \Gamma_{\alpha \beta}^{\alpha}=-\frac{1}{2}\left(\frac{e^{l_{\alpha}}+1}{e^{l_{\alpha}}-1}\right) \sec _{\alpha \beta, \beta}\left[\tan _{\beta, \alpha}^{2} \cos _{\alpha \beta, \alpha}-\sin _{\alpha \beta, \alpha} \tan _{\alpha \beta, \alpha}\right] .
\end{aligned}
$$

Repeating this entire procedure to equation (2), we obtain:

$$
\begin{aligned}
& \Gamma_{\alpha \alpha}^{\beta}=0, \\
& \Gamma_{\beta \beta}^{\beta}=-\frac{1}{2}\left(\frac{e^{l_{\beta}}+1}{e^{l_{\beta}}+1}\right) \tan _{\alpha, \beta}^{2}, \\
& \Gamma_{\alpha \beta}^{\beta}=\frac{1}{2}\left(\frac{e^{l_{\beta}}+1}{e^{l_{\beta}}-1}\right) \sec _{\alpha \beta, \alpha}\left[\tan _{\alpha \beta, \alpha}^{2} \cos _{\alpha \beta, \beta}-\sin _{\alpha \beta, \beta} \tan _{\alpha \beta, \beta}\right] .
\end{aligned}
$$

Substituting into (1) and (2) we obtain:

For an earthquake path $E(t)=\left(l_{\alpha}(t), l_{\beta}(t)\right)$,

$$
\begin{aligned}
\frac{d^{2} l_{\alpha}}{d t^{2}}= & \frac{1}{2}\left(\frac{e^{l_{\alpha}}+1}{e^{l_{\alpha}}-1}\right)\left[\tan _{\beta, \alpha}^{2}(t)\left(\frac{d l_{\alpha}}{d t}\right)^{2}-\left(\operatorname { s e c } _ { \alpha \beta , \beta } ( t ) \left[\tan _{\beta, \alpha}^{2}(t) \cos _{\alpha \beta, \alpha}(t)\right.\right.\right. \\
& \left.\left.\left.-\sin _{\alpha \beta, \alpha}(t) \tan _{\alpha \beta, \alpha}(t)\right]\right)\right]\left(\frac{d l_{\alpha}}{d t}\right)\left(\frac{d l_{\beta}}{d t}\right)
\end{aligned}
$$




$$
\begin{aligned}
\frac{d^{2}{ }_{\beta}}{d t^{2}}= & \frac{1}{2}\left(\frac{e^{l_{\beta}}+1}{e^{l_{\beta}}-1}\right)\left[\tan _{\alpha, \beta}^{2}(t)\left(\frac{d l_{\beta}}{d t}\right)^{2}-\left(\operatorname { s e c } _ { \alpha \beta , \alpha } ( t ) \left[\tan _{\alpha, \beta}^{2}(t) \cos _{\alpha \beta, \alpha}(t)\right.\right.\right. \\
& \left.\left.-\sin _{\alpha \beta, \beta}(t) \tan _{\alpha \beta, \beta}(t)\right)\right]\left(\frac{d l_{\alpha}}{d t}\right)\left(\frac{d l_{\beta}}{d t}\right)
\end{aligned}
$$
facts:

We now compute $\tan _{\alpha, \beta}(t)$ in terms of $l_{\alpha}(t)$ and $l_{\beta}(t)$. We need a few elementary

Lemma A. ([ $\left.\mathbf{W}_{2}\right]$.) Let the axis of two hyperbolic Mobius transformations $A, B$ intersect at a point $p$. Let $\theta$ be the angle at p formed by the segments along the axes of $A$ and $B$ to their respective attractive fixed points. Then

$$
\cos \theta=-\frac{\operatorname{sgn}(\operatorname{tr} A \operatorname{tr} B)(\operatorname{tr} A \operatorname{tr} B-2 \operatorname{tr} A B)}{\left(\operatorname{tr}^{2} A-4\right)^{1 / 2}\left(\operatorname{tr}^{2} B-4\right)^{1 / 2}} .
$$

LemMA B. Let $R$ be uniformized by $\Gamma$. For $A \in \Gamma$, let $\alpha$ be the closed geodesic in $R$ onto which the axis of $A$ projects. Then

$$
|\operatorname{tr} A|=2 \cosh \left(\frac{l(\alpha)}{2}\right), \quad l(\alpha)=\text { length of } \alpha \text { on } R .
$$

THEOREM C. ([FN, KE].) A once punctured torus is uniformized by a Fuchsian group $\Gamma \approx\langle A, B| B^{-1} A^{-1} B A$ is parabolic $\rangle$.

Let $x=\operatorname{tr} A, y=\operatorname{tr} B, z=\operatorname{tr} A B$. Then

(a) the quantities $x, y, z$ satisfy $x^{2}+y^{2}+z^{2}=x y z$ and $x, y, z>2$. Moreover,

(b) the Teichmüller space $\mathscr{T}_{1,1}=\left\{x^{2}+y^{2}+z^{2}=x y z\right\} \cap\{x, y, z>2\}$.

LEMMA 2.9.

$$
\tan ^{2} \theta(t)=\frac{1}{\cosh ^{2}\left(\frac{l_{\alpha}(t)}{2}\right) \sinh ^{2}\left(\frac{l_{\beta}(t)}{2}\right)-\cosh ^{2}\left(\frac{l_{\beta}(t)}{2}\right)} .
$$

Proof. Solving the expression in Theorem $C$ for $z=\operatorname{tr} A B$ and substituting into the expression in Lemma $\mathrm{A}$, we obtain

$$
\cos ^{2} \theta=\frac{\operatorname{tr}^{2} A \operatorname{tr}^{2} B-4\left(\operatorname{tr}^{2} A+\operatorname{tr}^{2} B\right)}{\left(\operatorname{tr}^{2} A-4\right)\left(\operatorname{tr}^{2} B-4\right)}
$$

Applying Lemma B, we obtain

$$
\cos ^{2} \theta=\frac{\cosh ^{2}\left(\frac{l_{\alpha}}{2}\right) \cosh ^{2}\left(\frac{l_{\beta}}{2}\right)-\left(\cosh ^{2}\left(\frac{l_{\alpha}}{2}\right)+\cosh ^{2}\left(\frac{l_{\beta}}{2}\right)\right)}{\left(\cosh ^{2}\left(\frac{l_{\alpha}}{2}\right)-1\right)\left(\cosh ^{2}\left(\frac{l_{\beta}}{2}\right)-1\right)},
$$

$$
\begin{aligned}
\tan ^{2} \theta & =\frac{1-\cos ^{2} \theta}{\cos ^{2} \theta} \\
& =\frac{\sinh ^{2}\left(\frac{l_{\alpha}}{2}\right) \sinh ^{2}\left(\frac{l_{\beta}}{2}\right)-\cosh ^{2}\left(\frac{l_{\alpha}}{2}\right) \cosh ^{2}\left(\frac{l_{\beta}}{2}\right)+\cosh ^{2}\left(\frac{l_{\alpha}}{2}\right)+\cosh ^{2}\left(\frac{l_{\beta}}{2}\right)}{\cosh ^{2}\left(\frac{l_{\alpha}}{2}\right) \cosh ^{2}\left(\frac{l_{\beta}}{2}\right)-\cosh ^{2}\left(\frac{l_{\alpha}}{2}\right)-\cosh ^{2}\left(\frac{l_{\beta}}{2}\right)} .
\end{aligned}
$$


After applying elementary hyperbolic trigonometric indentities, the denominator may be written as

$$
\cosh ^{2}\left(l_{\alpha} / 2\right) \sinh ^{2}\left(l_{\beta} / 2\right)-\cosh ^{2}\left(l_{\beta} / 2\right),
$$

and the numerator becomes equal to 1 .

Now consider a twist along $\alpha$. Since $d l \alpha / \mathrm{d} t=0$, the differential equations (7) and (8) for the path in $\mathscr{T}_{1,1}$ reduce to:

$$
\begin{gathered}
\frac{d^{2} \beta}{d t^{2}}=f\left(l_{\alpha}, l_{\beta}(t)\right)\left(\frac{d l_{\beta}}{d t}\right)^{2}, \text { where } \\
f\left(l_{\alpha}, l_{\beta}(t)\right)=\frac{1}{2} \operatorname{coth}\left(\frac{l_{\beta}(t)}{2}\right) \frac{1}{\cosh ^{2}\left(l_{\alpha} / 2\right) \sinh ^{2}\left(l_{\beta}(t) / 2\right)-\cosh ^{2}\left(l_{\beta}(t) / 2\right)} .
\end{gathered}
$$

Remark. A simple calculation shows that $f$ has one singularity when $l_{\beta}(t)=l_{\beta}^{\alpha} \equiv$ $2 \operatorname{coth}^{-1}\left[\cosh \left(l_{\alpha} / 2\right)\right]$ and $f$ is positive for $l_{\beta}(t)>l_{\beta}^{\alpha}$. Curiously enough, in [WW], the authors show that $l_{\beta}^{\alpha}$ is the smallest value of $l_{\beta}$ allowed on any surface with $l_{\alpha}$ fixed.

LemMA 2.10. Around $l_{\beta}(t)=l_{\beta}^{\alpha}$,

$$
f\left(l_{\alpha}, l_{\beta}(t)\right)=\frac{1}{2} \sinh ^{2}\left(l_{\alpha} / 2\right)\left(1 /\left(l_{\beta}(t)-l_{\beta}^{\alpha}\right)\right)+O(1) .
$$

Proof. Just expand in the obvious way.

LEMMA 2.11. If $l_{\beta}(t)$ is a solution to equation $(9)$, then $l_{\beta}(t) \rightarrow l_{\beta}^{\alpha}$ implies $i_{\beta}(t) \rightarrow 0$.

Proof. Rewrite equation (9) as

$$
\ddot{l}_{\beta}(t) / \dot{l}_{\beta}(t)=f\left(l_{\alpha}, l_{\beta}(t)\right) \dot{l}_{\beta}(t) .
$$

By the previous lemma, near $l_{\beta}(t)=l_{\beta}^{\alpha}$,

$$
f\left(l_{\alpha}, l_{\beta}(t)\right)=O\left(\frac{1}{l_{\beta}(t)-l_{\beta}^{\alpha}}\right),
$$

hence

$$
\frac{\ddot{l}_{\beta}(t)}{\dot{l}_{\beta}(t)}=O\left(\frac{1}{l_{\beta}(t)-l_{\beta}^{\alpha}}\right) \dot{l}_{\beta}(t)
$$

Integrating, we obtain:

$$
\log \left|\frac{l_{\beta}(t)}{l_{\beta}(0)}\right|=O\left(\log \left|\frac{l_{\beta}(t)-l_{\beta}^{\alpha}}{l_{\beta}(0)-l_{\beta}^{\alpha}}\right|\right) .
$$

The results follows immediately.

Define the Lyapunov potential $V\left(l_{\beta}, l_{\beta}\right)=l_{\beta} \cdot l_{\beta}$. Along a solution curve of equation (9), we have

$$
\dot{v}\left(l_{\beta}, \dot{l}_{\beta}\right)-l_{\beta} \ddot{l}_{\beta}+\left(\dot{l}_{\beta}\right)^{2}-l_{\beta}\left(f\left(l_{\alpha}, l_{\beta}\right) \dot{l}_{\beta}^{2}\right)+\dot{l}_{\beta}^{2}-\dot{l}_{\beta}^{2}\left(l_{\beta} f\left(l_{\alpha}, l_{\beta}\right)+1\right) .
$$

Since $f\left(l_{\alpha}, l_{\beta}\right)>0$, we see that $\dot{V}\left(l_{\beta}, l_{\beta}\right)>0$ along every solution curve.

It is clear that any constant function is a solution to (9), so by the fundamental uniqueness theorem of ODEs [CL], no solution starting below the $l_{\beta}$-axis $\left(l_{\beta}(0)<0\right)$ can cross the $l_{\beta}$-axis anywhere but at $l_{\beta}=l_{\beta}^{\alpha}$. 


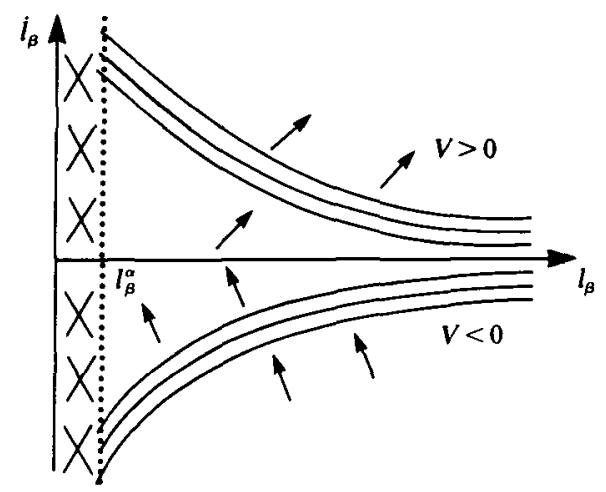

Figure 4. Curves of constant potential.

We will now show that any solution with $i_{\beta}(0)<0$, which hits $l_{\beta}^{\alpha}$ must do so in infinite time. Since $\dot{V}\left(l_{\beta}, l_{\beta}\right)>0$ along all solution curves, this will imply that any solution with $i_{\beta}(0)<0$ must approach a constant solution as $t \nearrow \infty$.

Proposition 2.12. Suppose $l_{\beta}(t)$ is a solution to (9). Then if $\frac{1}{2} \sinh \left(l_{\alpha} / 2\right)>1$, e.g., $l_{\alpha}>2.29, l_{\beta}(t)$ cannot reach $l_{\beta}^{\alpha}$ in finite time.

Proof. From Lemma 2.10 , near $l_{\beta}=l_{\beta}^{\alpha}$, equation (9) may be written

$$
\ddot{l}_{\beta}(t)=\left[\frac{1}{2} \sinh \left(\frac{l_{\alpha}}{2}\right) \frac{1}{l_{\beta}(t)-l_{\beta}^{\alpha}}+O(1)\right] i_{\beta}^{2}(t) .
$$

Let $c(\alpha)=\frac{1}{2} \sinh \left(l_{\alpha} / 2\right)>1$ (by hypothesis). Assuming $i_{\beta}(t) \neq 0$ for $t \in(a, b)$, we may divide both sides of equation (10) by $l_{\beta}(t)$. Integrating, we obtain

$$
\log \left|\frac{l_{\beta}(t)}{i_{\beta}(0)}\right|=\log \left(\frac{l_{\beta}(t)-l_{\beta}^{\alpha}}{l_{\beta}(0)-l_{\beta}^{\alpha}}\right) c(\alpha)+O\left(l_{\beta}(t)-l_{\beta}^{\alpha}\right)+c_{1}
$$

or

$$
\left|\frac{l_{\beta}(t)}{\dot{l}_{\beta}(0)}\right|=\frac{l_{\beta}(t)-l_{\beta}^{\alpha}}{l_{\beta}(0)-l_{\beta}^{\alpha}} c(\alpha) e^{c_{1}} e^{o\left(l_{\beta}(t)-l_{\beta}^{\alpha}\right)}
$$

Integrating again, we obtain:

$$
l_{\beta}(t)-l_{\beta}^{\alpha}=\left[\frac{(1-c(\alpha))\left|\dot{l}_{\beta}(0)\right| \operatorname{sgn}\left(i_{\beta}(t)\right)}{\left(l_{\beta}(0)-l_{\beta}^{\alpha}\right)^{c(\alpha)}} e^{c_{1}} \int_{0}^{t} e^{0\left(l_{\beta}(s)-l_{\beta}^{\alpha}\right)} d s\right]^{1 /(1-c(\alpha))}
$$

It is clear that the only way $l_{\beta}(t) \rightarrow l_{\beta}^{\alpha}$ is for $l_{\beta}(t)<0$, as $t \nearrow \infty$.

We have just shown that any solution to $(8)$ with $l_{\beta}(0)<0$ must approach a constant solution as $t \nearrow \infty$. This clearly contradicts the following theorem of Kerckhoff:

THEOREM. ([Kerckhoff, $\left.K_{1}\right]$ ) The Geodesic Length Function $l_{\gamma}$ of a simple closed curve $\gamma$ is convex along any earthquake path $E(R, \mu, t)$. It is strictly convex if $i(\gamma, \mu) \neq 0$.

We conclude, $\mathscr{E}$ is not $C^{2}$ on $\mathscr{T}_{1,1}$. 
Part $(b)$. We need the following theorem:

THEOREM ([FN, KE].) A one-holded torus is uniformized by a Fuchsian group

$$
\left.\Gamma \approx\langle A, B| B^{-1} A^{-1} B A \text { is hyperbolic }\right\rangle .
$$

Let $x=\operatorname{tr} A, y=\operatorname{tr} B, z=\operatorname{tr} A B, k=\operatorname{tr}\left(B^{-1} A^{-1} B A\right)$. Then

(a) the quantities $x, y, z$ and $k$ satisfy:

$$
k=x^{2}+y^{2}+z^{2}-x y z-2<-2 \text { and } x, y, z,-k>2 \text {. }
$$

Moreover,

(b) The Techmüller space

$$
\mathscr{T}_{1,0,1}=\left\{k=x^{2}+y^{2}+z^{2}-x y z-2\right\} \cap\{x, y, z,-k>2\} .
$$

By complete analogy with the case of the once punctured torus, we find that the ODEs for the earthquake along $\alpha$ reduce to:

$$
\begin{aligned}
\frac{d^{2} l_{\beta}}{d t^{2}}= & \frac{1}{2} \operatorname{coth}\left(\frac{l_{\beta}(t)}{2}\right)(1-4(k+2)) \\
& \times\left[\frac{1}{\cosh ^{2}\left(l_{\alpha} / 2\right) \sinh ^{2}\left(l_{\beta}(t) / 2\right)-\cosh ^{2}\left(l_{\beta} / 2\right)+4(k+2)}\right]\left(\frac{d l_{\beta}}{d t}\right)^{2} .
\end{aligned}
$$

This ODE has exactly the same asymptotic behavior as equation (8), hence $\mathscr{E}$ is not $C^{2}$ on $\mathscr{T}_{1,0,1}$.

Part (c). Given a Riemann surface of genus $g \geq 2$, choose coordinate curves containing $\alpha$ and $\beta$ in a handle.

It is a fundamental fact about twist deformations [FN] that if one twists along $\alpha$, the lengths of all curves that do not intersect $\alpha$ remain fixed. Apply part (b) to this handle.

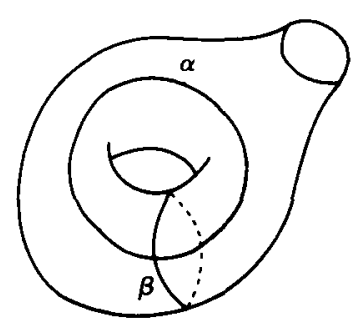

Figure 5. One holed torus.

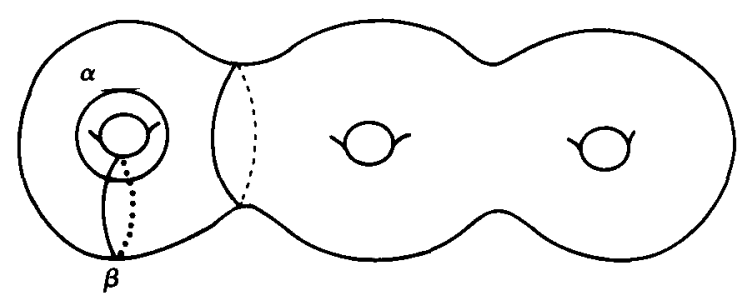

FIGURE 6 
The following is an immediate consequence of this theorem:

COROLlARY 2.13. The earthquake flow is not the geodesic flow for any Riemannian metric.

\section{Interesting problems}

(1) Find a Finsler metric whose geodesics are earthquake paths.

(2) Can the earthquake flow be reparametrized to make it a smooth flow?

(3) Is the infinitesimal generator for the earthquake flow Lipchitz?

\section{Acknowledgment}

This work was partially supported by Sloan Foundation Doctoral Dissertation Fellowship.

\section{REFERENCES}

[AH] L. V. Ahlfors. Lectures on Quasiconformal Mappings. Van Nostrand: New York, NY, 1966.

[BE] A. Beardon. The Geometry Discrete Groups. Springer Verlag: New York N.Y., 1983.

[CL] E. Coddington \& N. Levinson. Theory of Ordinary Differential Equations. McGraw Hill: New York, NY, 1955.

[FN] W. Fenchel \& J. Nielsen. Discontinuous groups of non-Euclidean motions. Unpublished manuscript.

[GKM] D. Gromoll, W. Klingenberg \& W. Meyer. Riemannsche Geometrie in Grossen, Lecture Notes in Mathematics 55. Springer-Verlag: NY, 1967.

[KE] L. Keen. A Rough Fundamental Domain for Teichmüller Spaces. Bull. Amer. Math. Soc. 83 (6) (1977), 1190-1226.

[K $K_{1}$ S. Kerckhoff. The Nielsen Realization Problem. Ann. of Math. 117 (1983), 235-265.

$\left[K_{2}\right]$ S. Kerckhoff. Earthquakes are Analytic. Comment. Math. Helv. 60 (1985).

$\left[\mathbf{K}_{\mathbf{3}}\right] \quad$ S. Kerckhoff. Lines of Minima in Teichmüller Space, To appear.

[ML] J. Milnor. Morse Theory. Ann Math. Studies 51. Princeton Univ. Press: Princeton, NJ, 1983.

[MOR] J. Morgan. Columbia University Lecture Notes.

[SP] M. Spivak. A Comprehensive Introduction to Differential Geometry, Vol. 1-5, Publish or Perish. Berkeley: CA 1970, 1975.

[T] W. P. Thurston. The Geometry and Topology of 3-Manifolds. Princeton University Lecture Notes.

$\left[\mathbf{W}_{1}\right] \quad S$. Wolpert. University of Maryland Lecture Notes.

$\left[W_{2}\right]$ S. Wolpert. On the symplectic geometry of deformation of a hyperbolic surface. Ann. of Math. 117 (1983), 207-234.

[WW] P. Waterman \& S. Wolpert. Earthquake Tessellations of Teichmüller Space. Trans. Amer. Math. Soc. 275 (1983), 157-167. 